\title{
有机发光二极管器件白光实现及其色坐标计算
}

\author{
王光华 ${ }^{1,2}$ ，张 杰 $^{1}$ ，高思博 ${ }^{1}$ ，段良飞 ${ }^{1}$ ，武艳鸣 ${ }^{1,2}$ ，周 芳 $^{1}$, \\ 尹 莉 ${ }^{1}$, 段登辉 ${ }^{1}$, 王丽琼 ${ }^{1}$, 季华夏 ${ }^{1}$
}

(1. 云南北方奥雷德光电科技股份有限公司, 云南 昆明 650223; 2. 昆明物理研究所, 云南 昆明 65022)

\begin{abstract}
摘要: 白光有机发光二极管可以作为照明、全彩色显示器的光源, 成为显示领域研发的重点方向。但 白光有机发光二极管的实现还存在性能控制较难, 色坐标易漂移等问题。本文通过有机发光二极管器 件白光实现及其色坐标计算, 初步获得实现白光 OLED 器件红、绿、蓝三基色的优化比, 通过实验验 证制备了色坐标为 $(0.31,0.35)$ 的白光 OLED 器件, 进一步通过理论计算和实验优化, 减少红光掺杂 浓度和增加绿光掺杂比例, 实现接近标准白光 $(0.33,0.33)$ 的有机发光二极管器件制备。
\end{abstract}

关键词: 有机发光器件; 白光; 色坐标计算; 器件制备

中图分类号：TN312.8 文献标识码：A 文章编号：1001-8891(2020)10-0958-05

\section{White Light Realization and Color Coordinate Calculation of OLED Devices}

\author{
WANG Guanghua ${ }^{1,2}$, ZHANG Jie ${ }^{1}$, GAO Sibo ${ }^{1}$, DUAN Liangfei ${ }^{1}$, WU Yanming ${ }^{1,2}$, \\ ZHOU Fang ${ }^{1}$, YIN LI ${ }^{1}$, DUAN Denghui ${ }^{1}$, WANG Liqiong ${ }^{1}$, JI Huaxia ${ }^{1}$ \\ (1. Yunnan Olightek Opto-electronic Technology Co., Ltd., Kunming 650223, China; \\ 2. Kunming Institute of Physics, Kunming 650223, China)
}

\begin{abstract}
White-light OLED devices can be used as a surface light source and for full color displays, which have become the focus of OLED research and development. However, there are some shortcomings in the realization of white-light OLED devices, such as insufficient luminous performance, difficult process control, and easy color drift. To solve these problems, the optimized ratios of primary colors(red, green, and blue) of organic OLED devices were preliminarily obtained through the realization of white light and calculation of the color coordinate. An experimental white-light OLED device was prepared with a color coordinate of $(0.31,0.35)$. Further, theoretical calculations and experimental optimizations were carried out to reduce the red-light doping concentration and increase the green-light doping ratio to realize standard white-light $(0.33$, 0.33) OLED devices.
\end{abstract}

Key words: OLED, white light, coordinate calculation, device preparation

\section{0 引言}

有机发光二极管 OLED（ organic light-emitting diode）具有自发光、厚度薄、响应时间快、可作为柔 性显示等优异特性, 被公认为最具发展潜力的固体照 明和显示器件 ${ }^{[1-2]}$ 。白光有机发光二极管 WOLED (white organic light-emitting diode) 既可作为平面照明 光源, 又可与彩色过滤层结合实现全彩色显示, 具有
广阔的应用前景 ${ }^{[3-4]}$ 。目前, 有机发光二极管通常采用 红、绿、蓝共掺杂的单发光层结构, 红、绿、蓝分别 掺杂的多发光层结构和通过下转换方式的光致发光 结构实现白光发射 ${ }^{[5-6]}$ 。其中, 共掺杂单发光层结构的 白光 OLED 器件易于设计与制造, 但器件光电性能较 依赖于高性能有机材料, 而且器件效率较低。通过下 转换实现白光显示的发光结构, 器件光电性能严重依 赖蓝光材料光电特性, 同时器件寿命较低。多发光层 
的叠层 OLED 结构器件驱动电压较高 ${ }^{[7-9]}$, 器件色坐 标会随着驱动电压发生漂移, 影响了器件实际应用。 为了获得性能稳定、高效的白光 OLED 器件, 通过掺 杂以多种颜色混合实现标准白光 OLED 器件制备, 主 要方法是利用互补色蓝色和黄色、红色和青色或红、 绿、蓝三基色混合等得到白光 ${ }^{[10-13]}$ 。为了提高试验效 率, 准确找到实现白光的红、绿、蓝三基色的优化比, 实现对有机发光二极管器件白光色坐标计算, 将有助 于为白光 $(0.33,0.33)$ OLED 器件的制备及其掺杂浓度 准确控制提供理论依据。

\section{1 获得白光所需要的三基色亮度比值计算}

有机发光实现白色显示的方法主要有以下几种: 由红、绿、蓝混合实现, 颜色匹配可用方程: $C(\mathrm{C})=X(\mathrm{X})$ $+Y(\mathrm{Y})+Z(\mathrm{Z})$ 来计算。其中, $(\mathrm{C})$ 为白色单位; $(\mathrm{X}) 、(\mathrm{Y})$ 、 $(Z)$ 为红、绿、蓝单位; $C$ 为白色数量; $X 、 Y 、 Z$ 为红、 绿、蓝数量。

白光三刺激值计算:

$$
\begin{gathered}
X=X_{1}+X_{2}+X_{3} \\
Y=Y_{1}+Y_{2}+Y_{3} \\
Z=Z_{1}+Z_{2}+Z_{3}
\end{gathered}
$$

式中: $X_{1} 、 X_{2} 、 X_{3}$ 为红色三刺激值; $Y_{1} 、 Y_{2} 、 Y_{3}$ 为绿 色三刺激值; $Z_{1} 、 Z_{2} 、 Z_{3}$ 为蓝色三刺激值。 $x_{1} 、 y_{1} 、 z_{1}$ 为红基色坐标; $x_{2} 、 y_{2} 、 z_{2}$ 为绿基色坐标; $x_{3} 、 y_{3} 、 z_{3}$ 为蓝基色色坐标; $L_{1} 、 L_{2} 、 L_{3}$ 为红、绿、蓝三基色亮 度, 白光的三刺激值可用公式(2)计算:

$$
\begin{gathered}
X=\frac{x}{y} L \\
Y=L \\
Z=\frac{Z}{y} L=\frac{1-x-y}{y} L
\end{gathered}
$$

将公式(2)式代入公式(1)可得:

$$
\begin{gathered}
X=\frac{x_{1}}{y_{1}} L_{1}+\frac{x_{2}}{y_{2}} L_{2}+\frac{x_{3}}{y_{3}} L_{3} \\
Y=L_{1}+L_{2}+L_{3} \\
Z=\frac{1-x_{1}-y_{1}}{y_{1}} L_{1}+\frac{1-x_{2}-y_{2}}{y_{2}} L_{2}+\frac{1-x_{3}-y_{3}}{y_{3}} L_{3}
\end{gathered}
$$

色坐标为:

$$
\begin{aligned}
& x=\frac{X}{X+Y+Z} \\
& y=\frac{Y}{X+Y+Z} \\
& z=\frac{Z}{X+Y+Z}
\end{aligned}
$$

由于 $x+y+z=1$, 因此通常用 $(x, y)$ 来表示色坐 标。标准白光的色坐标为 $(0.33,0.33)$ 。

将(3)式代入(4)式得:

$$
\begin{gathered}
x=\frac{\frac{x_{1}}{y_{1}} L_{1}+\frac{x_{2}}{y_{2}} L_{2}+\frac{x_{3}}{y_{3}} L_{3}}{\frac{1}{y_{1}} L_{1}+\frac{1}{y_{2}} L_{2}+\frac{1}{y_{3}} L_{3}}=0.33 \\
y=\frac{L_{1}+L_{2}+L_{3}}{\frac{1}{y_{1}} L_{1}+\frac{1}{y_{2}} L_{2}+\frac{1}{y_{3}} L_{3}}=0.33
\end{gathered}
$$

通过式(5)可以计算得到 OLED 器件红、绿、蓝、 三基色亮度之间的比例, 可根据比例调整三基色的发 光强度。另外通过式(5)计算得到理论三基色亮度之间 的比值, 但由于发光层产生的光要经过电子注入层、 电子传输层、密封层, 最后从阴极射出, 不同材料对 不同波长的光吸收情况不同, 所以如果按照(5)式计算 得到的三基色亮度比例进行掺杂时色坐标可能存在 偏离。

\section{2 光谱色品坐标计算}

要计算颜色的色品坐标, 需要先求得颜色的三刺 激值, 公式为:

$$
\begin{array}{ll}
X=k \int_{\lambda} \varphi(\lambda) \bar{x}(\lambda) \mathrm{d} \lambda & X_{10}=k_{10} \int_{\lambda} \varphi(\lambda) \bar{X}_{10}(\lambda) \mathrm{d} \lambda \\
Y=k \int_{\lambda} \varphi(\lambda) \bar{y}(\lambda) \mathrm{d} \lambda & Y_{10}=k_{10} \int_{\lambda} \varphi(\lambda) \bar{y}_{10}(\lambda) \mathrm{d} \lambda \\
Z=k \int_{\lambda} \varphi(\lambda) \bar{z}(\lambda) \mathrm{d} \lambda & Z_{10}=k_{10} \int_{\lambda} \varphi(\lambda) \bar{Z}_{10}(\lambda) \mathrm{d} \lambda
\end{array}
$$

式中: 积分范围 380 780 nm。在计算中应用了求和 近似积分的处理方法, 式(6)化为:

$$
\begin{array}{ll}
X=k \sum \varphi(\lambda) \bar{x}(\lambda) \Delta \lambda & X_{10}=k_{10} \sum \varphi(\lambda) \bar{x}_{10}(\lambda) \Delta \lambda \\
Y=k \sum \varphi(\lambda) \bar{y}(\lambda) \Delta \lambda & Y_{10}=k_{10} \sum \varphi(\lambda) \bar{y}_{10}(\lambda) \Delta \lambda(7) \\
Z=k \sum \varphi(\lambda) \bar{z}(\lambda) \Delta \lambda & Z_{10}=k_{10} \sum \varphi(\lambda) \bar{z}_{10}(\lambda) \Delta \lambda
\end{array}
$$

式中: $\varphi(\lambda)$ 称为颜色刺激函数。因为 OLED 器件是自 发光体, $\varphi(\lambda)$ 为 OLED 器件是自发光体辐射的相对光 谱功能分布:

$$
\varphi(\lambda)=S(\lambda)
$$

式(7)中: $\bar{x} 、 \bar{y} 、 \bar{z} 、 \bar{x}_{10} 、 \bar{y}_{10} 、 \bar{z}_{10}$ 为标准色度观察 的三刺激值。人眼观察的视角为 $1^{\circ} \sim 4^{\circ}$ 时采用 $\bar{x} 、 \bar{y}$ 、 $\bar{Z}$, 当 $4^{\circ} \sim 10^{\circ}$ 之间则采用 $\bar{x}_{10} 、 \bar{y}_{10} 、 \bar{z}_{10}$ 。本实验采 用前者, 图 1 为 1931 CIE 标准光谱三刺激值。常数 $k$ 
和 $k_{10}$ 称作归一化系数。

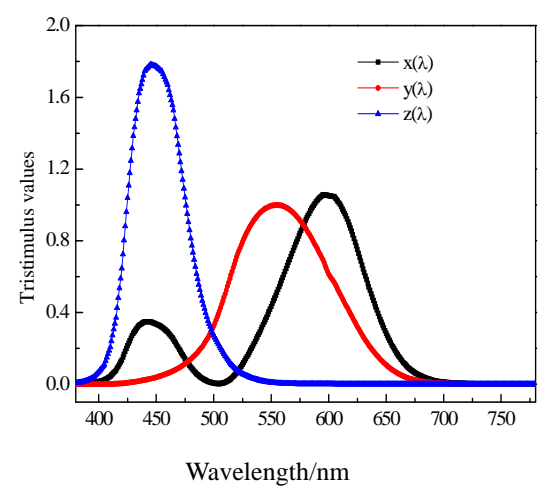

图 1 1931CIE 标准色度观察者光谱三刺激值

Fig.1 Spectral tristimulus values of 1931 CIE standard colorimetry

由式(8)计算出 OLED 器件三刺激值后, 由式(9) 计算出色品坐标值:

$$
\begin{array}{ll}
x=\frac{X}{X+Y+Z} & x_{10}=\frac{X_{10}}{X_{10}+Y_{10}+Z_{10}} \\
y=\frac{X}{X+Y+Z} & y_{10}=\frac{Y_{10}}{X_{10}+Y_{10}+Z_{10}} \\
z=\frac{X}{X+Y+Z} & y_{10}=\frac{Z_{10}}{X_{10}+Y_{10}+Z_{10}}
\end{array}
$$

图 2 和图 3 为在 $20 \mathrm{~mA} / \mathrm{cm}^{2}$ 电流密度下, 测试得 到的 OLED 器件光谱辐射值和归一化电致发光谱。将 测试得到相对光谱功率值代入上述公式, 可计算得到 OLED 色坐标值。其理论计算和实验测试结果如表 1 所示。从试验情况来看, 理论计算得到的色坐标与实 验测试结果相吻合。

\section{3 实现白光 $(0.33,0.33)$ 所需三基色发光比例 分析}

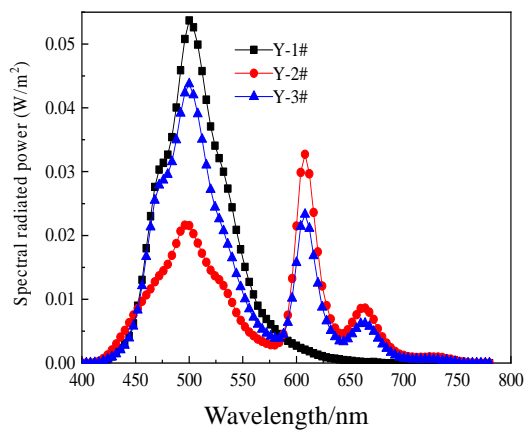

图 2 OLED 器件光谱辐射值

Fig.2 Light power radiation value of OLED devices

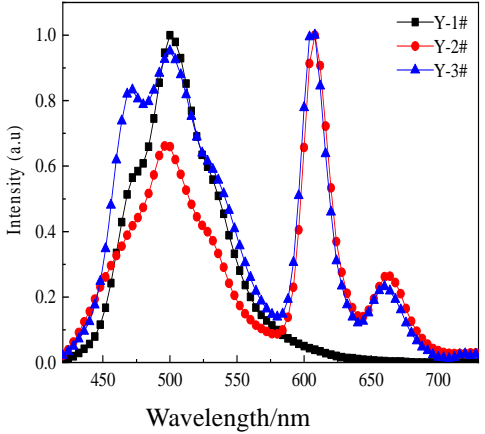

图 3 OLED 器件归一化 EL 光谱

Fig.3 Normalized electroluminescent spectrum of OLED devices

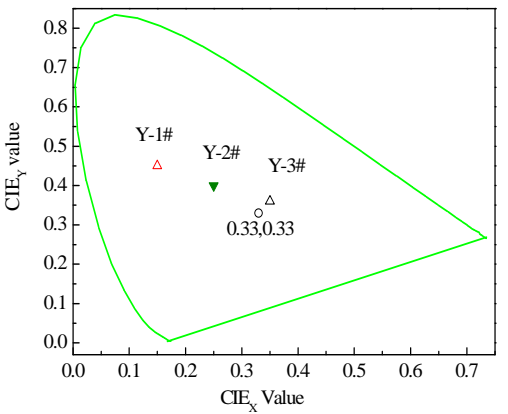

图 4 OLED 器件色品坐标计算结果

Fig.4 Calculated chromaticity coordinates of OLED devices 


\section{表 2 三基色发光强度比例计算}

Table 2 The calculated ratio of trichromatic luminescence intensity

\begin{tabular}{|c|c|c|c|c|c|}
\hline & \multicolumn{2}{|c|}{$\begin{array}{l}\text { Integral area of normalized } \\
\text { electroluminescence spectra }\end{array}$} & \multirow{2}{*}{$\begin{array}{l}\text { Required ratio of trichromatic luminance } \\
\text { of white OLED devices }\end{array}$} & \multicolumn{2}{|c|}{ Calculated coordinate values } \\
\hline & Area(square) & Percentage & & $\mathrm{CIE}_{X}$ & $\mathrm{CIE}_{Y}$ \\
\hline Blue+green & 80.95 & $69.63 \%$ & $71.2 \%$ & 0.15 & 0.37 \\
\hline Red & 35.31 & $30.37 \%$ & $28.8 \%$ & 0.35 & 0.65 \\
\hline White & 116.26 & $100 \%$ & $100 \%$ & 0.31 & 0.35 \\
\hline
\end{tabular}

现为蓝光 + 绿光亮度不够, 而红光亮度较大, 这是导 致色坐标有偏离的本质原因。实验中通过进一步减少 红光掺杂浓度或进一步增加绿光掺杂比例, 可以有效 改善发光强度之间配比关系, 实现接近白光 $(0.33$, 0.33)的白光 OLED 器件制备。

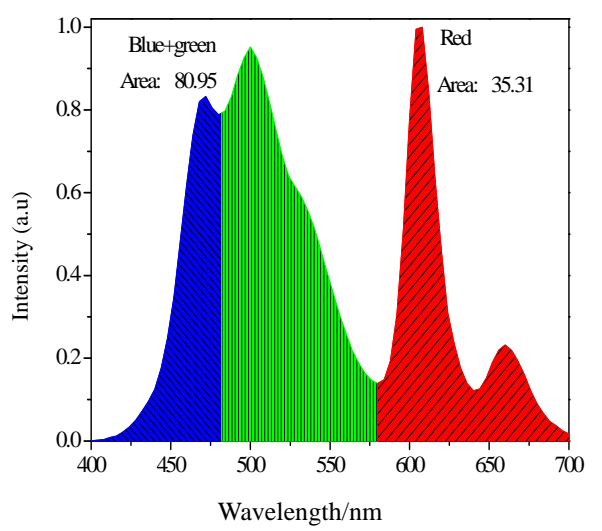

图 5 白光 OLED 器件 EL 光谱

Fig.5 Normalized electroluminescent spectrum of white OLED devices

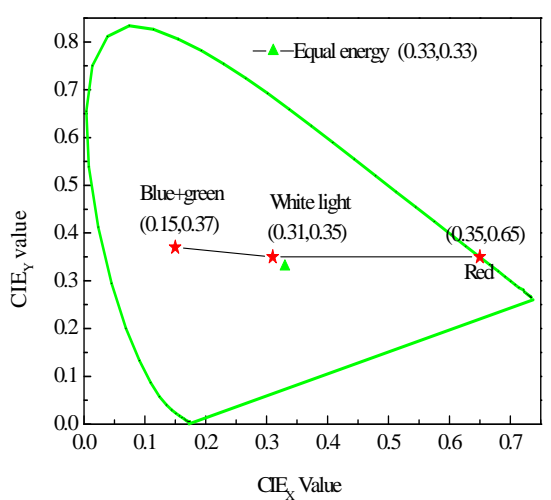

图 6 白光 OLED 器件色坐标计算值

Fig.6 The Calculated chromaticity coordinate of white OLED devices

\section{4 结论}

实验通过三基色亮度比值计算, 得到白光 OLED 器件红、绿、蓝三基色亮度之间的理论比例, 并与光
谱色品坐标计算得到的色坐标与实验测试结果相吻 合。根据计算得到的三基色混合形成白光所需比例, 计算得到红光、绿光、蓝光所需亮度比例分别为 28.8\%、56.8\%、15.4\%。依据理论计算结果，实验制 备了白光 OLED 器件, 色坐标为 $(0.31,0.35)$, 还稍微 偏离白光等能点 $(0.33,0.33)$ 。理论计算与实验结果的 偏离, 主要是由于发光层产生的光要经过电子注入 层、电子传输层、密封层, 最后从阴极射出, 不同材 料对不同波长的光其吸收情况不同。同时，计算得到 三基色发光强度比例偏离理论计算值，蓝光十绿光比 例为 $69.63 \%$, 小于理论计算值的 $71.2 \%$, 而红光比例 $30.37 \%$ ，大于理论计算值的 $28.8 \%$ 。实验可以通过进 一步减少红光掺杂浓度或进一步增加绿光掺杂比例, 改善发光强度之间配比关系, 实现接近色坐标为 $(0.33,0.33)$ 的白光 OLED 器件制备。

\section{参考文献:}

[1] 王旭鹏, 密保秀, 高志强, 等. 白光有机发光器件的研究进展[J]. 物 理学报, 2011, 60(8): 843-857.

WANG Xupeng, MI Baoxiu, GAO Zhiqiang, et al. Progress of white organic light-emitting device[J]. Acta Physica Sinica, 2011, 60(8): 843-857.

[2] 刘佰全, 高栋雨, 王剑斌, 等. 白光有机发光二极管的研究进展 [J]. 物理化学学报, 2015, 31(10): 1823-1852.

LIU Baiquan, GAO Dongyu, WANG Jianbin, et al. Progress of white organic light-emitting diodes[J]. Acta Physico-Chimica Sinica, 2015, 31(10): 1823-1852.

[3] 王光华, 季华夏, 张篖丹, 等. 阴极耦合层对顶发射白光 OLED 器件 光电性能的影响 $[\mathrm{J}]$. 液晶与显示, 2015, 30(3): 437-443.

WANG Guanghua, JI Huaxia, ZHANG Xiaodan, et al. Effect of outcouping layers on optical and electrical properties of top-emitting organic white light emitting devices[J]. Chinese Journal of Liquid Crystals and Displays, 2015, 30(3): 437-443.

[4] ZHANG Xiaowen, MO Bingjie, LIU Liming, et al. Highly efficient bilayer-structure yellow-green OLED with MADN hole-transport layer and the impedance spectroscopy analysis[J]. Spectroscopy and Spectral 
Analysis, 2015, 35(12): 3296-3299.

[5] LIU Yu, Masayoshi Nishiura. $\pi$-conjugated aromatic enynes as a single-emitting component for white electroluminescence[J]. J. Am. Chem. Soc., 2006, 17(128): 5592-5593.

[6] Jou Jwohuei, WANG Chunjan, LIN Yupu. Color-stable efficient fluorescent pure-white organic light-emitting diodes with device architecture preventing excessive exciton formation on guest[J]. Applied Physics Letters, 2008, 92: 223504.

[7] WANG Yu, HUA Yulin, WU Xiaoming. High-efficiency fluorescent white organic light-emitting device with double emissive layers[J]. Organic Electronics, 2008, 9(2): 273-278.

[8] ZHANG Zhiqiang, WANG Qi, DAI Yanfeng. High efficiency fluorescent white organic light-emitting diodes with red, green and blue separately monochromatic emission layers[J]. Organic Electronics, 2009, 10(3): 491-495.

[9] Jonghee Lee, Joo-Won Lee, Nam Sung Cho, et al. Highly efficient all phosphorescent white organic light-emitting diodes for solid state lighting applications[J]. Current Applied Physics, 2014(14): 84-87.

[10] 马颖, 韩薇, 张方辉, 等. 发光层混合掺杂的白光 OLED 器件 [J]. 液 晶与显示, 2011, 26(1): 40-43.

MA Ying, HAN Wei, ZHANG Fanghui, et al. White organic light -emitting diodes based on mixed doping emitting layer[J]. Chinese Journal of Liquid Crystals and Displays, 2011, 26(1): 40-43.

[11] CHANG Chihhao, CHENG Hsingchieh, LU Yinjui. Enhancing color gamut of white OLED displays by using microcavity green pixels[J]. Elsevier, 2010, 11(2): 247-254

[12] JOU Jwohuei, CHOU Yichieh, SHEN Shiming. High-efficiency very -high color rendering white organic light-emitting diode with a high triplet interlayer[J]. Journal of Materials Chemistry, 2011, 21(46): $18523-18526$

[13] WANG Guanghua, ZHAO Huiqiong, DENG Rongbin, et al. Research on the preparation and chromaticity coordinates shift mechanism of organic white light top-emitting devices[J]. Spectrosc. Spectr. Anal, 2016, 36(11): 3758-376 